\title{
Coexistence of Intrahepatic Cholestasis of Pregnancy Accompanied by Hepatitis B Flare in a Pregnant Woman and the Subsequent Development of Postpartum Thyroiditis and HBS Antigen Loss: A Case Report
}

\author{
Huseyin Begenik ${ }^{\mathrm{a}, \mathrm{c}}$, Ahmet Cumhur Dulger ${ }^{\mathrm{b}}$, Ramazan Esen ${ }^{\mathrm{a}}$, Habib Emre ${ }^{\mathrm{a}}$
}

\begin{abstract}
A 23-year-old pregnant woman was admitted to our hospital due to pruritus and right upper quadrant pain. Her abdominal ultrasound revealed hepatomegaly and the fetal ultrasonography was normal. Her HBS antigen was positive and $\mathrm{HBe}$ antigen was negative. HBV-DNA level was 350,000 copies/ml. Coincidental hepatobiliary diseases in pregnancy were ruled out. The patient received a diagnosis of HBe antigen negative hepatitis B and tenofovir disoproxil $245 \mathrm{mg}$ /day was prescribed. At a follow-up visit one month later, she reported no improvement in her pruritus. The levels of liver transaminases were near-normal and HBV DNA was zero. However, her biochemistry revealed raised alkaline phosphatase and gamma glutamyl transferase levels. Her consecutively calculated fasting serum bile acid levels were too high. A diagnosis of severe intrahepatic cholestasis of pregnancy associated with hepatitis $\mathrm{B}$ infection was made and a treatment of ursodeoxycholic acid 1000 $\mathrm{mg}$ /day was prescribed while she continued to take tenofovir disoproxil. She had a normal delivery and there were no adverse outcomes for the fetus. Several weeks later, she was readmitted with tremor and nervousness. Her TSH level was low and CRP level was high. The rest of the laboratory findings were normal and an ultrasonography of thyroid showed paranchymal heterogenity. The aggregate of findings was consistent with postpartum thyroiditis. Finally, L-thyroxine was initiated on a daily basis. Interestingly, at the week of 96, loss of Hepatitis B surface antigen was detected during tenofovir treatment.
\end{abstract}

Keywords: Intrahepatic cholestasis of pregnancy; Hepatitis B; Postpartum thyroiditis

\footnotetext{
Manuscript accepted for publication February 23, 2012

${ }^{a}$ Yuzuncu Yil University, Departmant of Gastroenterology, Van, Turkey

${ }^{b}$ Yuzuncu Yil University, Departmant of Internal Medicine, Van, Turkey

${ }^{\mathrm{c}}$ Corresponding author: Huseyin Begenik, Yuzuncu Yil University,

Departmant of Internal Medicine, Van, Turkey.

Email: hbegenik@hotmail.com
}

doi:10.4021/jmc594w

\section{Introduction}

Many diseases affect the liver during pregnancy, the most frequent being preeclampsia, eclampsia, acute fatty liver of pregnancy, HELLP syndrome, hepatic rupture, hyperemesis gravidarum and intrahepatic cholestasis of pregnancy. Moreover, liver test abnormalities may exist as a result of many diseases that occur coincidentally during pregnancy. The laboratory findings of each of these disorders are usually similar, ranging from elevated liver-related enzymes and cholestasis [1]. Intrahepatic cholestasis of pregnancy (IHCP) is a rare disorder with a prevalence of one in $1000-10,000$ pregnancies. It is usually defined as cholestasis without other causes of biliary obstruction and liver dysfunction during the third trimester and, as a rule, it normally resolves following delivery [2]. The ethiology of this disease has not been well established. One possible explanation is a gene variant of hepatocanalicular transport proteins and their regulators which affect the estrogen and progesterone mechanism [3, 4].

The diagnosis of IHCP is based on evidence of elevated serum bile acid concentrations $(>11 \mathrm{mmol} / \mathrm{L})$ and decreased glycine/taurine bile acid ratio (below 1.0) in connection with cholestasis and pruritis during the third trimester of pregnancy [5]. To confirm the diagnosis, the coexistant diseases (e.g., viral hepatitis, autoimmune cholangitis, biliary tract disorders and systemic diseases) or medication use (e.g., herbal medicine) characterized by cholestasis should be promptly ruled out [6]. HBe antigen negative pregnant patients with positive HBs antigen should be followed closely by means of ALT and HBV DNA testing and treated with antiviral agents once confirmation of hepatitis B flare during hypertransaminasemia is established [7].

Postpartum thyroiditis (PPT) is a destructive autoimmune thyroiditis that begins within the first few months after delivery, $85 \%$ of patients with PPT have positive antithyroid antibodies. The thyrotoxic phase typically begins primarily and may be followed by a hypothyroid phase. Abnormalities in thyroid function tests resolve in most patients [8]. Very rarely, a flare of hepatitis B may be associated with IHCP. In such cases, a prompt diagnosis can be made hardly. Here we report the coexistence of IHCP with hepatitis B infection and 
the subsequent development of PPT and loss of hepatitis B antigen in a woman.

\section{Case Report}

A 29-year-old pregnant woman was referred to our hospital because of elevated liver-related enzymes. A diagnosis of hepatitis B had been made seven years earlier and she did not receive any therapy for Hepatitis B. She did not smoke, drink alcohol or use herbal medicine. The patient was born and lived in eastern Turkey. This was her first pregnancy and there was no history of abortion. Her mother had pregnancyrelated cholestasis and died in her 60s. On physical examination, the vital signs were normal except for mild tenderness on her right upper quadrant with scleral subicterus.

Her blood pressure was $115 / 85 \mathrm{mmHg}$ and she had a pulse of 90 beats per minute at a temperature of $36.6^{\circ} \mathrm{C}$. The patient's hematologic values were within the normal limits. The laboratory results obtained were as follows: aspartate aminotransferase level, $125 \mathrm{U}$ per liter (normal range, 0 to 42); alanine aminotransferase level, $225 \mathrm{U}$ per liter (normal range, 0 to 47); alkaline phosphatase level, $700 \mathrm{U}$ per liter (normal range, 42 to 140); gamma glutamyl transferase level, $350 \mathrm{U}$ per liter (normal range, 5 to 60 ); and direct bilirubin level, $0.4 \mathrm{mg}$ per deciliter (normal range, 0.0 to $0.4 \mathrm{mg}$ per deciliter). The international normalized ratio (INR) was 1.0.

Her HBS antigen was positive but $\mathrm{HBe}$ and hepatitis delta antigens were negative. HBV-DNA level was 350,000 copies $/ \mathrm{ml}$. She tested negative for other causative agents of viral hepatitis. Autoimmune and metabolic liver disease markers were also negative. An ultrasonographic study of the abdomen revealed a viable fetus at 24 weeks of gestation and showed an enlarged liver which measured as $19 \mathrm{~mm}$; the appearance of the liver was homogeneous, without gallbladder stones or biliary dilation. The pancreas was normal and there was no splenomegaly. Moreover, MRCP revealed no abnormality in the entire biliary tract as well as pancreatic duct. Medication at discharge included tenofovir disoproxil $245 \mathrm{mg}$ /day with antipruritic skin gels. One month after discharge, she returned to the hepatology clinic due to worsening of pruritus especially during the night. When admitted for the second time, her HBV DNA level was negative but direct bilirubin levels as well as cholestatic enzymes were higher.

Her prothrombin time was normal. Serum bile acid concentration was measured as $47 \mathrm{mmol} / \mathrm{L}$. A diagnosis of IHCP was made and while tenofovir disoproxil was continued, a treatment with UDCA (15 mg/kg per day) and topical emollients was started. Two weeks later, at a follow-up visit, the patient was comfortable, and her pruritus was resolved. Furthermore, levels of the conjugated bilirubin and liver-related enzymes were also normalized. Following the fourth week of delivery, she returned to the hospital due to inability to sleep and tremors.

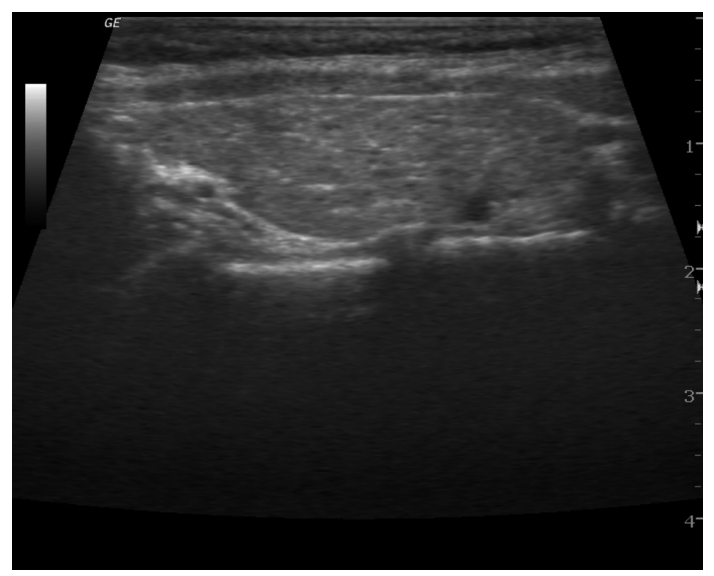

Figure 1. Paranchymal heterogenity on thyroid US.

Hormonal analysis revealed hyperthyroidism with higher CRP level and elevated titers of antithyroid antibodies. A thyroid ultrasonography showed paranchymal heterogeneity (Fig. 1). On the basis of these findings, postpartum thyroiditis was diagnosed and a short course of beta-blocker was started. Four months later, a subsequent hypothyroidal phase developed and $0.05 \mathrm{mg}$ of L-thyroxine daily was prescribed. Furthermore, 96 weeks later, loss of HBS antigen developed during tenofovir treatment. The patient is currently thriving in her normal medical state.

\section{Discussion}

The case reported had a rare combination of different diseases. We considered firstly liver disorders occurring coincidentally with pregnancy such as a delta coinfection or a severe exacerbation of hepatitis $\mathrm{B}$, since the patient had previously received a diagnosis of being a hepatitis $\mathrm{B}$ carrier, and the patient had HBs antigen and higher HBV DNA levels. Delta hepatitis was ruled out by a negative HDV RNA test. Diagnosing IHCP at the time of the patient's initial presentation was done hardly because the moderate cholestasis can be seen in biliary disorders but it could also reflect a cholestatic variety of viral hepatitis [9].

In this patient, co-morbidity, including autoimmune or metabolic liver diseases was also excluded by further biochemical and serologic methods. The therapy for hepatitis $\mathrm{B}$ in pregnant patients consists of nucleoside or nucleotide analogs. Tenofovir and telbivudine are listed by the FDA as pregnancy category B drugs [10], of which the most commonly available is tenofovir disoproxil at a dose of $245 \mathrm{mg} /$ day, which we used for the treatment of the discussed case.

Despite the evidence of treatment-related low levels of HBV DNA, moderately elevated transaminases and cholestatic enzymes and direct hyperbilirubinemia persisted and her pruritus worsened at the fifth week of the antiviral treat- 
ment. IHCP frequently causes cholestasis in the third trimester of pregnancy, with a mean presentation at 30 weeks gestation. Biochemical tests of patients typically show elevated levels of serum alkaline phosphatase (two to four times the normal level) and conjugated bilirubin $(<5 \mathrm{mg} / \mathrm{dL})$. Liver transaminases may also be elevated, but rarely higher than fourfold [11].

Elevated levels of total serum bile acid concentrations are present in almost all patients with IHCP [5] as was seen in our case. Ursodeoxycholic acid (UDCA; $10-20 \mathrm{mg} / \mathrm{kg}$ per day) is the first-line treatment for IHCP [12]. A cascade of persistent symptoms and biochemical features at the second admission were characteristic of IHCP, so we favored treatment with UDCA at a dose of $15 \mathrm{mg} / \mathrm{kg}$ per day. Symptomatic treatment for pruritus was also started. In this patient, reducing HBV DNA levels by maintaining tenofovir was a good therapeutic approach, so we continued the antiviral therapy.

PPT is an autoimmune disorder and develops within 2 to 6 months following delivery. Transient symptoms of hyperthyroidism may develop. High titers of antithyroid antibodies are almost always present. One of the hallmarks of PPT is a markedly depressed TSH level with a high free thyroxine level during the postpartum period. Hypothyroidism may develop in a minority of patients [13]. TSH is a useful tool to detect postpartum thyroiditis and should be measured where possible in all patients post pregnancy. Therapy with levothyroxine sodium is indicated for patients with thyroid failure [14].

Loss of serum HBS antigen is reported as final endpoint for the treatment of hepatitis B [15]. In HBe antigen negative patients, tenofovir-induced loss of HBS antigen at week 48 was reported as $0 \%$ [16]. At week 96 , she also had a loss of HBS antigen with antibody seroconversion against hepatitis B surface antigen. In light of the presented case report, tenofovir disoproxil should be considered for the treatment of $\mathrm{HBe}$ antigen negative pregnant patients.

IHCP can also occur in pregnant hepatitis B patients. A high index of suspicion is necessary in order to diagnose IHCP, particularly in pregnant women who have a flare of hepatitis B. Furthermore, there may be a causal relationship between the IHCP and postpartum thyroiditis. These two disorders may share the same autoimmune ethiology.

\section{References}

1. Doshi S, Zucker SD. Liver emergencies during pregnancy. Gastroenterol Clin North Am. 2003;32(4):12131227, ix.
2. Germain AM, Carvajal JA, Glasinovic JC, Kato CS, Williamson C. Intrahepatic cholestasis of pregnancy: an intriguing pregnancy-specific disorder. J Soc Gynecol Investig. 2002;9(1):10-14.

3. Poupon R. Intrahepatic cholestasis of pregnancy: from bedside to bench to bedside. Liver Int. 2005;25(3):467468.

4. Pauli-Magnus C, Lang T, Meier Y, Zodan-Marin T, Jung D, Breymann C, Zimmermann R, et al. Sequence analysis of bile salt export pump (ABCB11) and multidrug resistance p-glycoprotein 3 (ABCB4, MDR3) in patients with intrahepatic cholestasis of pregnancy. Pharmacogenetics. 2004;14(2):91-102.

5. Brites D. Intrahepatic cholestasis of pregnancy: changes in maternal-fetal bile acid balance and improvement by ursodeoxycholic acid. Ann Hepatol. 2002;1(1):20-28.

6. Fagan EA. Intrahepatic cholestasis of pregnancy. Clin Liver Dis. 1999;3(3):603-632.

7. Terrault NA, Jacobson IM. Treating chronic hepatitis B infection in patients who are pregnant or are undergoing immunosuppressive chemotherapy. Semin Liver Dis. 2007;27 Suppl 1:18-24.

8. Stagnaro-Green A. Postpartum thyroiditis. Best Practice \& Research Clinical Endocrinology \& Metabolism. 2004 Vol. 18, No. 2, pp. 303-316.

9. European Association for the Study of the L. EASL Clinical Practice Guidelines: management of cholestatic liver diseases. J Hepatol. 2009;51(2):237-267.

10. EASL Clinical Practice Guidelines. Management of chronic hepatitis B. Journal of Hepatology. 2009; 50:237-38.

11. Knox TA, Olans LB. Liver disease in pregnancy. N Engl J Med. 1996;335(8):569-576.

12. Lammert F, Marschall HU, Matern S. Intrahepatic Cholestasis of Pregnancy. Curr Treat Options Gastroenterol. 2003;6(2):123-132.

13. Walfish PG, Meyerson J, Provias JP, Vargas MT, Papsin FR. Prevalence and characteristics of post-partum thyroid dysfunction: results of a survey from Toronto, Canada. J Endocrinol Invest. 1992;15(4):265-272.

14. Lazarus JH. Clinical manifestations of postpartum thyroid disease. Thyroid. 1999;9(7):685-689.

15. Yuen MF, Wong DK, Yuan HJ, Sum SM, Lai CL. HBsAg seroclearance in Chinese patients receiving lamivudine therapy for chronic hepatitis B virus infection. $\mathrm{J}$ Clin Microbiol. 2004;42(10):4882-4884.

16. Marcellin P, Heathcote EJ, Buti M, Gane E, de Man RA, Krastev Z, Germanidis G, et al. Tenofovir disoproxil fumarate versus adefovir dipivoxil for chronic hepatitis B. N Engl J Med. 2008;359(23):2442-2455. 